\title{
Aggregation of Health Assessment Indicators of Industrial Systems
}

\author{
Ludovic Rizzolo ${ }^{1}$ Bouthaina Abichou ${ }^{1}$ Alexandre Voisin ${ }^{1}$ Naïm Kosayyer $^{2}$ \\ ${ }^{1}$ Centre de Recherche en Automatique de Nancy (CRAN), Nancy-Université, CNRS, \\ Boulevard des Aiguillettes B.P. 70239, 54506 Vandoeuvres-lès-Nancy, France \\ ${ }^{2}$ PREDICT, 19 avenue de la Forêt de Haye, CS10508, 54519, Vandoeuvre-lès-Nancy, France \\ ludovic.rizzolo@cran.uhp-nancy.fr, bouthaina.abichou@cran.uhp-nancy.fr, alexandre.voisin@cran.uhp-nancy.fr,
} naim.kosayyer@predict.fr

\begin{abstract}
In the field of Prognostics and Health Management (P.H.M.), Health Monitoring can be seen as the first step to manage the global health state of complex systems. Health Monitoring of industrial systems focuses on accurately describing the health state of a system, using several equipment indicators. However, managers and maintainers have to make decisions. Such decisions can be hard to make while watching all indicators of the system simultaneously. In order to ease the decision making process, a synthetic indicator, which represent the actual system's state, can be used. In this paper, we will present an approach for building an aggregated indicator characterizing the global health state of a system. This approach was implemented on the TELMA platform (integrated TELeMAintenance platform for research and education) which simulates an industrial process.
\end{abstract}

Keywords: P.H.M, Health Assessment, Industrial Systems, Utility Functions, Aggregation, Choquet Integral.

\section{Introduction}

In the field of Prognostics and Health Management, Health Monitoring can be seen as the first step to manage global health state of complex systems [1]. Nevertheless, as monitoring systems are usually built around particular subsystems; they focus more on diagnostic of degradations and failures modes of these subsystems. Hence, they are managing a set of indicators focused on preventing failures of the system [2]. Thus, the operator, piloting or maintaining the system, has to consider a consequent number of indicators (performance and/or failure oriented) to make decision. The scientific added value of our approach is to complete the precedent view by adding a single global indicator to monitor the health state of a system. That is, by considering the system as a whole to observe and evaluate both its performances and drifts (usually representative of degradations). Therefore, this synthesis of information about the system will ease decision making [3], in a global manner. Nevertheless, investigation of all indicators, in details, will always be mandatory as it is already done.

This work has been done as a part of the BMCI project (Bilan de santé pour la Maintenance et la Conduite Intelligente: Health Assessment and MonitoringBased Intelligent Maintenance and Control), which aims at providing Health monitoring facilities for naval fleet Prognostics and Health Management. The facilities are developed as on-the-shelve components including: wireless sensors, acquisition, processing and visualization systems at level of equipment, ship and fleet. Its major innovation lies in the monitoring of degradations rather than failures, as present alarm monitoring systems (AMS) do. Moreover, this project will respond to the ship's operators need of a more synthetic view of the ship health in order to pilot or maintain her.

In the next section, we will present the characteristics and constitution of health assessment indicators. Then, we will focus on how to aggregate these indicators into a value representative of the system's health. The last section will illustrate the application and compare different aggregation operators.

\section{Health Assessment}

Health assessment is composed of a set of indicators describing a detailed view of a system or equipment. However, while managing or maintaining, a too large number of indicators can hinder the efficiency of decisions and operations. So, it seems to be appropriate to deliver a synthesis of the indicators, constituting the health assessment, in the form of a single value standing for a global evaluation.

\subsection{Health Assessment's indicators}

Several methods exist to define indicators characterizing the health of a system [4][5]. These approaches are mainly based on functional and dysfunctional models, of the systems considered, to help identifying these indicators.

\subsubsection{Functional model based indicators}

These indicators are mainly focused on representing performances of the system in terms of production and/or effectiveness and efficiency. They are determined by use of a functional analysis of the system considered (e.g. SADT: Structured Analysis and Design Technique, IDEF0: Integration Definition for Function Modeling). The goal is to characterize, on one hand, the flows of the function, and on the other hand, its performances. One can find in this second category especially: the effectiveness (ratio of results to objectives), and the efficiency (ratio of results to engaged resources). 
The indicators are defined according to the properties of main input and output flows of the function considered (e.g. Figure 1) and/or more synthetic data (energetic effectiveness, deviation from objectives).

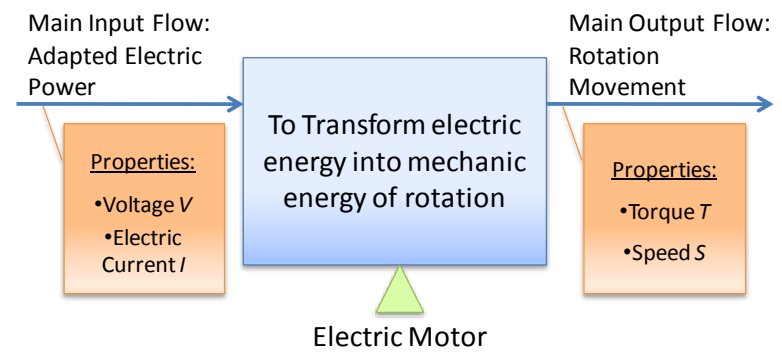

Figure 1: Example of functional model based indicators

\subsubsection{Dysfunctional model based indicators}

Dysfunctional indicators are mainly intended to maintenance purpose. These indicators are meant, on one hand, to report the occurrence of degradations and failures, and on the other hand, to help maintenance operations.

A first category is based on dysfunctional analysis of the system (e.g. FMECA/HAZOP: Failure Mode Effects and Criticality Analysis/HAZard OPerability), and report the occurrence of the degradation modes. The processing of these dysfunctional analyses (FME$\mathrm{CA} / \mathrm{HAZOP}$ ) makes possible to determine the indicators linked to these degradations.

In a second category, one can find, for example, aging indicators (Real age and Virtual age) allowing to complement the general health state analysis. Indeed, considering two identical systems of equal performances, but one being older than the other, it is obvious that the less old system would be preferred to the older one. The virtual age indicator mentioned above is intended to assess the impact of the equipment's operations on the aging process (e.g. the number of starts and accelerations will "age" more quickly a motor). The value of this indicator is, by definition, greater than or equal to the real age.

\subsection{Health State}

To ease the decision making process, of piloting or maintaining, the set of health assessment's indicators could be synthesized/aggregated in a more global single indicator, called health state. This indicator represents a snapshot of the system's health in comparison to a reference. Therefore, an aggregation operator is needed. This operator has to model the importance of indicators in comparison to the chosen reference (which could be given by the manufacturer of the system, an expert, or an observation period) and potentially take into account the interactions between them (which can be seen as an added source of information about the system's state).

\section{Aggregation process}

Aggregation is a process synthesizing in a global value (an aggregated value) the information coming from var- ious sources (which could be from various entities, points of view...). This aggregated value must satisfy some preferences of a group of individuals or some properties, thus helping decision making in order to achieve a consensus [6]. In our context, aggregation must represent, at best, the health state of the system based on the previously mentioned indicators.

These indicators being of different natures and scales, the process of aggregation is split in 2 steps: normalization and aggregation.

\subsection{Normalization}

This first step of normalization is mandatory so that the whole set of indicators: (1) are on a same scale of values (2) expressing an equivalent semantic. These two points make possible for the aggregation operator to work on a set of values of equivalent scale and semantic.

In order to help decision making, datas have to be transformed into information. For that purpose, requirements about working performances can be used (given, for instance, by expert knowledge). For example, the only torque data of 6 N.m does not inform on a motor performances. However, if one knows, as a requirement, that, this same motor, has to deliver a torque value between 4 and 7 N.m ; then, gathering both data and requirement, allows to give a relevant information: the torque is within the target. Moreover, Normalization makes possible to compare different indicators (e.g. a torque and an electric current or the output speed of a motor and the output speed of a speed reducer). Normalization is, therefore, mandatory.

We will use for this purpose utility functions [7]. Utility functions translate the relevance of a value in regard to a referential value and/or an objective. Therefore, it will express a correspondence between the value of a system indicator and the health of this system.

For each indicator $I_{i}$ the utility function $u_{i}: \mathbb{R} \rightarrow[0,1]$ $u_{i}: \mathbb{R} \rightarrow[0,1]$ is defined by:

$$
u_{i}=f\left(I_{i}\right)
$$

There are several ways to define these functions [7]:

1. Using data resulting from experience feedback. A history of indicators data taken during identified working period (good behavior, degradation mode) makes possible to identify range of values or specific working point linked to working modes. The drawback of this method is that it requires a learning period during which it is mandatory that phases of good and bad behavior are identified.

2. Using requirements from expert knowledge on the system. These requirements define critical values for which the system will not be able to match expectations required. It is possible to define directly, with the expert, "ideals" ranges of values or working points. The drawback of this method is that the expert knowledge is not always sufficient to define quantitatively these utility function's characteristics values.

We decided use both methods simultaneously. In that way, a less consequent experience feedback is required 
which allows identifying the general shape of the function and an estimation of its boundaries. The expert knowledge allows precising this estimation and adding additional constraints along with validating the system's observations. In particular, it makes possible to dismiss chosen sets of values out of requirement (for example because of an imprecise identification of good behavior working mode) and to add knowledge where data are missing (because the whole space of system's states cannot be explored during learning period).

Dysfunctional indicators of aging present a different kind of normalization. For these indicators, we will consider the period between two scheduled maintenance operations. Thus, when this operation has just been made, the utility is equal to 1 ; and the more close the date of the next operation is, the more decreases the utility. Therefore, the loss of confidence one can have in a system more subject to failure (because of a distant scheduled maintenance) is assessed.

\subsection{Aggregation operator}

Several aggregation operators exist [8], for instance: mean type operators (arithmetic mean, weighted arithmetic mean) and O.W.A. (Ordered Weighted Averaging) [9] are among the most used.

O.W.A. is a particular operator where criteria's data are ordered in an increasing order and where weights are not assigned to specific criterion but to the rank in the order. Thus, using the weights vector, one can adjust O.W.A. weights to range between minimum and maximum operator.

Nevertheless, in our application, which aims at combining functional and dysfunctional information, interaction phenomenon will occurs. For instance, considering a diesel engine, filter clogging and fuel consumption stand for health indicators. A "bad" result on the first (a high level of clogging) implies a bad result on the second (increase of consumption). Thus, it is important to take into account interaction between indicators. This can be made using fuzzy integral operators [10].

The operator chosen is the Choquet integral. This operator is non linear and can be seen as a generalization of the weighted arithmetic mean or the ordered arithmetic average (O.W.A.) [10]. The distinctive characteristic of this operator is that it makes possible to take into consideration the interactions between criteria, thus being more relevant with our application. In that way, criteria in interaction stated as "synergy" will have a greater impact on (increase) the aggregated value (especially if they are of high values). Whereas, criteria in interaction stated as "redundancy" will have a lesser impact on this aggregated value (even if they are of high values). In case of no interactions between criteria, the integral become an ordered weighted average.

Let $\{X\}:\left\{x_{1}, \cdots, x_{n}\right\}$ be a set of normalized criteria, consider a capacity $\mu: \wp(X) \rightarrow[0,1]$ on this set, verifying:

$$
\left\{\begin{array}{c}
\mu(\varnothing)=0 \\
\mu(X)=1 \\
\mu(A) \leq \mu(B), \forall A \subseteq B \text { et } \forall B \subseteq X
\end{array}\right.
$$

Then the Choquet integral is defined as follows:

$$
C_{\mu}\left(x_{1}, \cdots, x_{n}\right)=\sum_{i=1}^{n} x_{(i)}\left[\mu\left(A_{(i)}\right)-\mu\left(A_{(i+1)}\right)\right]
$$

Where (.) is a permutation operator such that:

$$
\begin{aligned}
x_{(1)} & \leq \cdots \leq x_{(n)} \quad \text { and } \quad A_{(i)}:\left\{x_{(i)}, \cdots, x_{(n)}\right\} \quad \text { with } \\
A_{(n+1)} & =\emptyset
\end{aligned}
$$

For applying this integral, it is mandatory to identify the coefficients of the capacity. It can be seen as a set of coefficients, not only on a single criterion, but on a set of criteria as well. Each subset represents a possible combination of criteria. So, their number increases exponentially according to the number of criteria $\left(2^{n}-2\right.$ subsets for $n$ criteria). Hence, the identification of capacity's coefficients becomes a major concern when applying the Choquet integral.

It is possible to decrease the number of capacities coefficients by restraining the number of interacting criteria. For example, one can say that at most $k$ criteria are interacting. This kind of capacity is said to be $k$ additive [10].

Several methods identifying capacities, used when applying Choquet integral as an aggregation operator, exist [11]. These methods use mainly three kinds of inputs:

- Data sets of the system considered ordered according to the expert knowledge.

- Data set of the system considered marked according to the expert knowledge.

- Expert knowledge about criteria dealing with their interactions and importance.

The identification problem is, roughly speaking, an optimization problem [12][13]. The goal of this step is to minimize the difference between the results of the integral and the evaluations of the system's health (that the expert has through the indicators).

A first approach consists in asking the expert to give a partial preorder to the health assessment sets and/or to evaluate them. Then, some approaches will try to minimize the difference between global evaluations, given by the expert, and aggregated values given by the Choquet integral (with respect to constraints given by the expert on the interactions and importance of criteria) which results in finding the matching capacity. The major inconvenience of this method is that it is difficult for the expert to evaluate, by a global score, the state of the system in relation to the indicators chosen; this is why we decided not to use it.

Other approaches use only a partial preorder and constraints given by the expert. Using linear programming or looking to minimize the variance on the global scores, these methods result in the matching capacity. Their drawback is the use of data sets. Indeed, in order to have an enough representative basis of the expert's system view, it is mandatory to cover the whole space described by the indicators. Yet, even with an extensive learning period, all situations (or combination of indicators values) may not have been reached by the system. That case requires the creation of fabricated situations to cover the gaps in data sets. However, by adding these fabricated cases, a solution will be induced, which will not actually represent the expert's point of view. More- 
over, the situations present in data sets must be sufficiently differentiable for the expert to order them.

The chosen method was proposed by Grabisch [12]. The basic idea of this method is that without any knowledge of the system the best capacity to aggregate the indicators is additive (without interactions) and uniform (same importance for all criteria). Then, the goal is to minimize the distance between a uniform capacity and the aimed capacity (which verify the constraints given by the expert). Thus, the aggregation operator takes into account only the expert's point of view on interactions and importance of indicators.

In order to use this identification method, the expert's point of view on the system's state must be gathered. We will consider 2-additive capacities. It is, indeed, difficult for an expert to consider interactions between more than two criteria. Expert knowledge gathering will be done by asking questions like: Is this indicator more important than the others? Which indicators are of equal importance? Is it possible to quantify the importance of this indicator in relation to the others? (Is it twice as much important or more?) How these two indicators contribute together toward the global system's state (is it question of synergy, redundancy or is there no interaction)?

The expression of interaction and importance constraints, given by the expert, cannot easily be made on the capacity itself. So, we use the Möbius representation of a capacity $\mu($.$) [10] defined by:$

$$
m_{\mu}(B)=\sum_{A \subseteq B}(-1)^{b-a} \mu(A), \forall B \subseteq X
$$

Where $a$ and $b$ represent the cardinalities of the respective subsets $A$ and $B$.

This Möbius representation of a capacity verifies:

$$
\left\{\begin{array}{c}
m_{\mu}(\varnothing)=0, \sum_{A \subseteq X} m_{\mu}(A)=1 \\
\sum_{\substack{\forall A \subseteq B \\
\forall A \ni\{i\}}} m_{\mu}(A) \geq 0, \forall B \subseteq X, \forall i \in B
\end{array}\right.
$$

In terms of Möbius representation, the Choquet integral with respect to a capacity $\mu$ is defined by:

$$
C_{m_{\mu}}\left(x_{1}, \cdots, x_{n}\right)=\sum_{A \subseteq X} m_{\mu}(A) \bigwedge_{i \in A} x_{i}
$$

Where $\Lambda$ represent the minimum operator.

With this representation the capacity's coefficients of couples of indicators reflects the kind of interaction between them:

- no interactions: $m_{\mu}\left(\left\{x_{i}, x_{j}\right\}\right)=0$

- In case of synergy: $m_{\mu}\left(\left\{x_{i}, x_{j}\right\}\right)>0$

- In case of redundancy: $m_{\mu}\left(\left\{x_{i}, x_{j}\right\}\right)<0$

In the same way, the importance of a criterion cannot be quantified only by the coefficient of the singleton that it is associated with; one must consider every interaction between the criterion and the others. We use for this purpose the Shapley importance index, which takes into account the mean importance of a criterion in relation to every possible interactions of this criterion with all of the others. The Shapley importance index [10] is defined by:

$$
\phi_{m_{\mu}}\left(\left\{x_{i}\right\}\right)=\sum_{A \subseteq X \backslash\left\{x_{i}\right\}} \frac{1}{a+1} m_{\mu}\left(A \cup\left\{x_{i}\right\}\right)
$$

Similarly, it is possible to express constraints over importance of interactions with the interaction importance index [10] defined as follows:

$$
\begin{array}{r}
I_{m_{\mu}}\left(\left\{x_{i}, x_{j}\right\}\right) \\
=\sum_{A \subseteq X \backslash\left\{x_{i}, x_{j}\right\}} \frac{1}{a+1} m_{\mu}\left(A \cup\left\{x_{i}, x_{j}\right\}\right)
\end{array}
$$

As for an example, let us consider the following indicators in the scope of assessing the health of the function "To transform electrical energy into mechanical energy of rotation": Torque (To), Real Age (RA) and Virtual Age (VA) (equations of the example will use the number assigned to each indicator).

We find in this example one functional indicator (torque) and two dysfunctional indicators of aging.

Considering importance, the first statement that the expert can make is that if the functional indicator is "good" then global state of the system has to be "good" whatever the values of dysfunctional indicators are. Thus, the expert defines the torque as more important than the other indicators. A second statement can be that he wants to consider the two aging indicators in an equivalent way (because he thinks that if either one becomes "bad" then he cannot have enough confidence in the system because of a distant last scheduled maintenance operation). Therefore, he defines an equal importance for these two indicators. The following constraints express these considerations:

$$
\phi_{m_{\mu}}(\{\mathrm{To}\})>\phi_{m_{\mu}}(\{\mathrm{RA}\})=\phi_{m_{\mu}}(\{\mathrm{VA}\})
$$

In terms of interactions, the expert states that, considering the couple \{torque, real age \}, the information on these two indicators contributes to determine the global state of the system, thus defining a synergy. This means that, even if the score of real age is low (because last scheduled maintenance operation is distant), if the score of torque is "good", then the aggregated value must be "good". However, the expert does not find enough information while considering only the couple \{real age, virtual age \}, thus defining a redundancy (partly due to the fact that virtual age is constructed with some information of real age), leading to the following constraints:

$$
\left\{\begin{array}{l}
m_{\mu}(\{\text { To, RA }\})>0 \\
m_{\mu}(\{\text { To }, V A\})>0 \\
m_{\mu}(\{\text { RA, VA }\})<0
\end{array}\right.
$$

This ends this short example intended to illustrate how the constraints can be formalized from expert's knowledge. In the next section we will present a detailed application. 


\section{Application}

\subsection{Presentation of the TELMA platform}

\subsubsection{The system}

The TELMA platform simulates an industrial process of unwinding and stamping/cutting strip (metal strip for automotive industry, paper for printer/paper maker or cloth for textile). The system is composed of four functions: Bobbin Changing, Strip Accumulation, Stamping/Cutting and Advance (Figure 2).

The Bobbin Changing function executes a rotation between two bobbin when the current one is empty (The strip quantity of a bobbin is configurable and the amount of strip is measured by a sensor). The Strip Accumulation function unwinds and stocks the strip ("wave" shown on Figure 1). It is used in the industry, for instance for online strip ends joining operations or to absorb unwinding variations. The Stamping/Cutting function executes the operation of stamping/cutting. The Advance function carries the strip under the press.

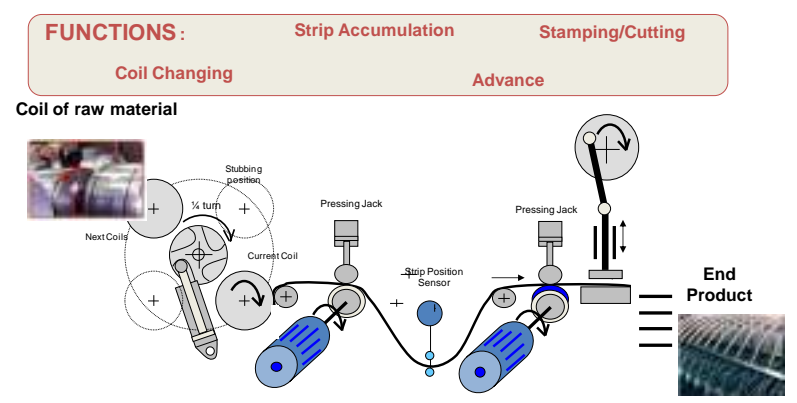

Figure 2: TELMA unwinding and stamping/cutting process

\subsubsection{The Strip Accumulation function}

The approach had been implemented on the Strip Accumulation function. This function is constituted of three sub-functions: To Transform electrical energy into mechanical energy of rotation (supported by an electric motor), To Transmit rotation (supported by a pulleybelt system) and To Transform rotation movement into linear movement (supported by a drum). A sensor is used to determine the stock level and, when there is not enough strip in the stock, the accumulation motor is activated until the stock is refilled. The acceleration control of the motor increases the speed of rotation according to its activation time (to a certain extend because the acceleration curve is " $\mathrm{S}$ " shaped).

\subsubsection{Indicators}

We focused on the sub-function To Transform electrical energy into mechanical energy of rotation. Table 1 presents the chosen indicators for health assessment.

\begin{tabular}{|c|c|c|c|}
\hline Class & Name & Unit & Id \\
\hline \multirow{4}{*}{ Functional } & Torque & $\mathrm{N} . \mathrm{m}$ & To \\
\cline { 2 - 4 } & Electric Current & $\mathrm{A}$ & $\mathrm{EC}$ \\
\cline { 2 - 4 } & Effectiveness & - & $\mathrm{Eff}$ \\
\cline { 2 - 4 } & Speed & $\mathrm{rd} / \mathrm{s}$ & $\mathrm{Sp}$ \\
\hline \multirow{3}{*}{ Dysfunctional } & Acceleration & $\mathrm{rd} / \mathrm{s}^{2}$ & $\mathrm{Acc}$ \\
\cline { 2 - 4 } & Real Age & $\mathrm{h}$ & $\mathrm{RA}$ \\
\cline { 2 - 4 } & Virtual Age & $\mathrm{h}$ & $\mathrm{VA}$ \\
\hline
\end{tabular}

Table 1: Indicators of the function: To transform electrical energy into mechanical energy

Functional indicators are properties of input or output flows of the motor (for example torque and speed are taken before reduction gear). As explained in 4.1.2, this motor is constantly starting and stopping to refill the stock of strip, so we decided to use acceleration as a dysfunctional indicator because of its sensitivity to any degradation. Real age is the working time since the last scheduled maintenance operation. Virtual age is based on the same time but take also into account the acceleration time and the starting torque ratio, resulting in a more important value.

\subsection{Normalization functions}

By applying the approach presented in section 3.1, we have determined normalization functions for the set of indicators previously proposed.

As explained previously, the distribution of values from a learning period allowed us to identify the general shape of these functions (we consider a range of utility equal to 1 for the support of the distribution). The expert adds constraints presented in Table 2

\begin{tabular}{|c|c|}
\hline Indicator & \multicolumn{1}{|c|}{ Constraints } \\
\hline Torque & $\begin{array}{l}\text { Torque is mandatory for the function } \\
\text { but if it is too low then it is of less utili- } \\
\text { ty. However it must not be higher than } \\
\text { the support of the distribution }\end{array}$ \\
\hline $\begin{array}{c}\text { Electric Cur- } \\
\text { rent }\end{array}$ & $\begin{array}{l}\text { Utility must match the support of the } \\
\text { distribution with only little variance }\end{array}$ \\
\hline Effectiveness & $\begin{array}{l}\text { Utility must match the support of the } \\
\text { distribution. }\end{array}$ \\
\hline Speed & $\begin{array}{l}\text { As long as there is speed the system } \\
\text { is operating, but if it is too low then it is } \\
\text { of less utility. A small variance is ac- } \\
\text { ceptable beyond the support of the dis- } \\
\text { tribution }\end{array}$ \\
\cline { 1 - 2 } Acceleration & $\begin{array}{l}\text { Values below the support of the dis- } \\
\text { tribution may be significant of degrada- } \\
\text { tion and can be considered of low utili- } \\
\text { ty. The variance beyond the support of } \\
\text { the distribution should be small, well } \\
\text { as, a too important value of acceleration } \\
\text { is clearly a sign of degradation. }\end{array}$ \\
\hline Real Age & $\begin{array}{l}\text { The maintenance operations are } \\
\text { scheduled every 150 hours of working } \\
\text { operation. We consider a reduction of } \\
\text { confidence after 135 hours. }\end{array}$ \\
\hline Virtual Age
\end{tabular}

Table 2: Constraints expressed by the expert for indicators normalization 
An example of these normalization functions can be seen on Figure 3. The two graphs show distribution of values of speed (above) and torque (below). Distributions are expressed in percentage of population (on the left scale) and the normalization function is represented by lines of a lighter color (with the corresponding utility value on the right scale).
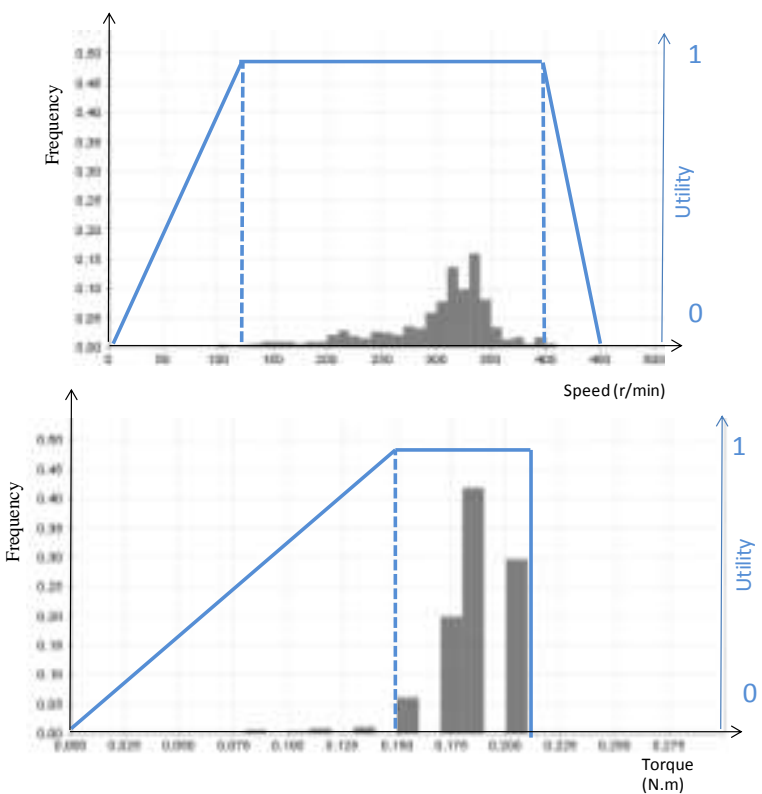

Figure 3: Example of normalization functions: speed (above) and torque (below)

\subsection{Aggregation operator}

\subsubsection{Constraints for capacity determination}

Table 3 and Table 4 present constraints expressed by the expert regarding the system's health state (We will use indicators Id presented in Table 1).

\begin{tabular}{|c|c|}
\hline $\begin{array}{c}\text { Importance } \\
\text { of indicator }\end{array}$ & $\begin{array}{c}\text { Importance } \\
\text { of interactions }\end{array}$ \\
\hline$\{$ To $\} \sim\{\mathrm{Sp}\}>\{\mathrm{EC}\}$ & $\{$ To,EC $\} \sim\{\mathrm{EC}, \mathrm{Sp}\}$ \\
$\{\mathrm{Acc}\}>\{\mathrm{RA}\} \sim\{\mathrm{VA}\}$ & $\{$ To,Eff $\} \sim\{\mathrm{Eff}, \mathrm{Sp}\}$ \\
$\{\mathrm{To}\}>\{\mathrm{Acc}\}$ & \\
\hline
\end{tabular}

Table 3: Constraints for capacity identification in terms of importance of indicators and interactions

\begin{tabular}{|l|l|l|l|l|l|l|}
\hline & EC & Eff & Sp & Acc & RA & VA \\
\hline To & Syn & Red & Syn & Syn & $\begin{array}{l}\text { Syn } \\
\text { Neut }\end{array}$ & $\begin{array}{l}\text { Syn } \\
\text { Neut }\end{array}$ \\
\hline EC & - & Red & Syn & Syn & $\emptyset$ & $\emptyset$ \\
\hline Eff & - & - & Red & $\begin{array}{l}\text { Red } \\
\text { Neut }\end{array}$ & $\varnothing$ & $\varnothing$ \\
\hline Sp & - & - & - & Red & $\begin{array}{l}\text { Syn } \\
\text { Neut }\end{array}$ & $\begin{array}{l}\text { Syn } \\
\text { Neut }\end{array}$ \\
\hline Acc & - & - & - & - & Syn & Syn \\
\hline RA & - & - & - & - & - & Red \\
\hline
\end{tabular}

Table 4: Constraints for capacity identification in terms of type of interactions
In Table 4 interactions of synergy type are marked as "Syn", interactions of redundancy are marked as "Red", absence of interactions are marked as "Neut" (for neutral) and absence of constraints given by the expert will be marked as $\emptyset$.

\subsubsection{Determined capacity}

The capacities identified using the Kappalab package of GNU R system are presented in Table 5.

\begin{tabular}{|c|c|}
\hline \multicolumn{2}{|c|}{ Capacities } \\
\hline$m_{\mu}(\{\mathrm{To}\})=0.0001$ & $m_{\mu}(\{\mathrm{EC}, \mathrm{Sp}\})=0.0000$ \\
$m_{\mu}(\{\mathrm{EC}\})=0.0167$ & $m_{\mu}(\{\mathrm{EC}, \mathrm{Acc}\})=0.0500$ \\
$m_{\mu}(\{\mathrm{Eff}\})=0.0424$ & $m_{\mu}(\{\mathrm{EC}, \mathrm{RA}\})=0.0000$ \\
$m_{\mu}(\{\mathrm{Sp}\})=0.1000$ & $m_{\mu}(\{\mathrm{EC}, \mathrm{VA}\})=0.0000$ \\
$m_{\mu}(\{\mathrm{Acc}\})=0.1000$ & $m_{\mu}(\{\mathrm{Eff}, \mathrm{Sp}\})=0.0000$ \\
$m_{\mu}(\{\mathrm{RA}\})=0.2000$ & $m_{\mu}(\{\mathrm{Eff}, \mathrm{Acc}\})=-0.0010$ \\
$m_{\mu}(\{\mathrm{VA}\})=0.2000$ & $m_{\mu}(\{\mathrm{Eff}, \mathrm{RA}\})=0.0000$ \\
$m_{\mu}(\{\mathrm{To}, \mathrm{EC}\})=0.0000$ & $m_{\mu}(\{\mathrm{Eff}, \mathrm{VA}\})=0.0000$ \\
$m_{\mu}(\{\mathrm{To}, \mathrm{Eff}\})=0.0000$ & $m_{\mu}(\{\mathrm{Sp}, \mathrm{Acc}\})=-0.1000$ \\
$m_{\mu}(\{\mathrm{To}, \mathrm{Sp}\})=0.4000$ & $m_{\mu}(\{\mathrm{Sp}, \mathrm{RA}\})=0.0000$ \\
$m_{\mu}(\{\mathrm{To}, \mathrm{Acc}\})=0.1000$ & $m_{\mu}(\{\mathrm{Sp}, \mathrm{VA}\})=0.0000$ \\
$m_{\mu}(\{\mathrm{To}, \mathrm{RA}\})=0.0000$ & $m_{\mu}(\{\mathrm{Acc}, \mathrm{RA}\})=0.0500$ \\
$m_{\mu}(\{\mathrm{To}, \mathrm{VA}\})=0.0000$ & $m_{\mu}(\{\mathrm{Acc}, \mathrm{VA}\})=0.0500$ \\
$m_{\mu}(\{\mathrm{EC}, \mathrm{Eff}\})=-0.0082$ & $m_{\mu}(\{\mathrm{RA}, \mathrm{VA}\})=-0.2000$ \\
\hline
\end{tabular}

Table 5: Identified capacity for health state

\subsubsection{Presentation of an example set}

We will now consider examples of health assessment vectors. In Tables 6 and 7 we will use examples Id, indicators Id and their normalized values.

\begin{tabular}{|l|l|}
\hline Id & Description \\
\hline E1 & $\begin{array}{l}\text { Torque and effectiveness are low because of a bear- } \\
\text { ing degradation. Dysfunctional indicators are good } \\
\text { (no big impact on speed) }\end{array}$ \\
\hline E2 & Degradation impacting speed \\
\hline E3 & $\begin{array}{l}\text { Same type of degradation as E2 but next scheduled } \\
\text { maintenance operation is closer (we think E2 and E3 } \\
\text { should have relatively close health state) }\end{array}$ \\
\hline E4 & $\begin{array}{l}\text { Overconsumption of current because of an electrical } \\
\text { degradation. Next scheduled maintenance operation } \\
\text { is distant }\end{array}$ \\
\hline E5 & $\begin{array}{l}\text { Following of E4. A lot of starts and accelerations to } \\
\text { compensate the degradation have fastened the aging } \\
\text { process. The idea is to compare E4 and E5. We think } \\
\text { we should find similar results. }\end{array}$ \\
\hline
\end{tabular}

Table 6: Description of example set

\begin{tabular}{|l|l|l|l|l|l|l|l|}
\hline Id & To & EC & Eff & Sp & Acc & RA & VA \\
\hline E1 & 0.20 & 0.80 & 0.60 & 0.90 & 0.90 & 1.00 & 1.00 \\
\hline E2 & 0.90 & 0.80 & 0.60 & 0.20 & 0.50 & 1.00 & 1.00 \\
\hline E3 & 0.90 & 0.80 & 0.60 & 0.40 & 0.65 & 0.40 & 0.20 \\
\hline E4 & 0.95 & 0.20 & 0.00 & 0.95 & 0.85 & 1.00 & 1.00 \\
\hline E5 & 0.95 & 0.20 & 0.00 & 0.95 & 0.85 & 0.80 & 0.10 \\
\hline
\end{tabular}

Table 7: Examples of health assessment (in term of normalized vectors) 


\subsubsection{Comparison of different aggregation operators}

In this sub-section we will compare health states of the previous example set obtained by different aggregation operators, in order to compare the results of the Choquet integral with known operator's. The two other operators are the arithmetic mean and O.W.A. and are defined respectively as follow on a set $X$ of $n$ indicators:

$$
\begin{gathered}
\bar{X}=\frac{1}{n} \sum_{i=1}^{n} x_{i} \\
\operatorname{OWA}\left(x_{1}, \cdots, x_{n}\right)=\sum_{i=1}^{n} w_{i} x_{(i)}
\end{gathered}
$$

Where (.) represent a reordering of the $x_{i}$ in a nonincreasing order as in (2).

In order to use the O.W.A. one must determine the set of weights $w_{i}$ to apply. The approach used was proposed by Yager [9] and was reviewed by $\mathrm{Xu}$ [14] as one of the main method to determined O.W.A. weights. This method is based on using linguistic quantifiers. The weights are given by:

$$
w_{i}=Q\left(\frac{i}{n}\right)-Q\left(\frac{i-1}{n}\right), \text { for } i=1, \cdots, n
$$

Where $Q$ is defined as follow by Zadeh [15]:

$$
Q(j)=\left\{\begin{array}{c}
0, \text { if } j<a \\
\frac{j-a}{b-a}, \text { if } a \leq j \leq b \\
1, \text { if } b<j
\end{array}\right.
$$

Where $(a, b)$ represent the non-decreasing proportional fuzzy linguistic quantifier. We chose it equal to $(0.3,0.8)$ to express that "most" of the indicators should be "good" to result in a "good" health state. This choice was motivated by the will to have an O.W.A. operator which is neither too pessimistic nor too optimistic. This leads to the following weights:

$$
\begin{array}{ll}
\text { - } w_{1}=0 & \text { - } w_{5}=0.2857 \\
\text { - } w_{2}=0 & \text { - } w_{6}=0.1714 \\
\text { - } w_{3}=0.2572 & \bullet w_{7}=0 \\
\text { - } w_{4}=0.2857 &
\end{array}
$$

\subsubsection{Application on an example}

We will apply the three operators on one example presented previously:

$$
\begin{aligned}
& \overline{\mathrm{X}}(\mathrm{E} 1)=\frac{0.2+0.8+0.6+0.9+0.9+1+1}{7} \approx 0.77 \\
& O W A(E 1)=0 * 1+0 * 1+0.2572 * 0.9+ \\
& 0.2857 * 0.9+0.2857 * 0.8+0.1714 * 0.6+0 * \\
& 0.2 \approx 0.82 \\
& C_{\mu}(E 1)=0.0001 * 0.2+0.0167 * 0.8+0.0424 * \\
& 0.6+0.1 * 0.9+0.1 * 0.9+0.2 * 1+0.2 * 1+0 * \\
& 0.2+0 * 0.2+0.4 * 0.2+0.1 * 0.2+0 * 0.2+0 * \\
& 0.2-0.0082 * 0.6+0 * 0.8+0.05 * 0.8+0 * 0.8+
\end{aligned}
$$

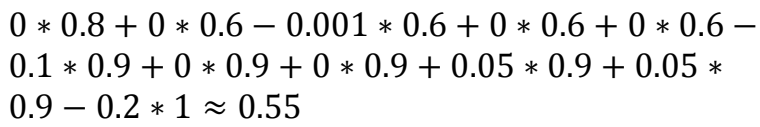

\subsubsection{Comparison of Health States}

Figure 4 will compare health states for the set of examples using the previous aggregation operators.

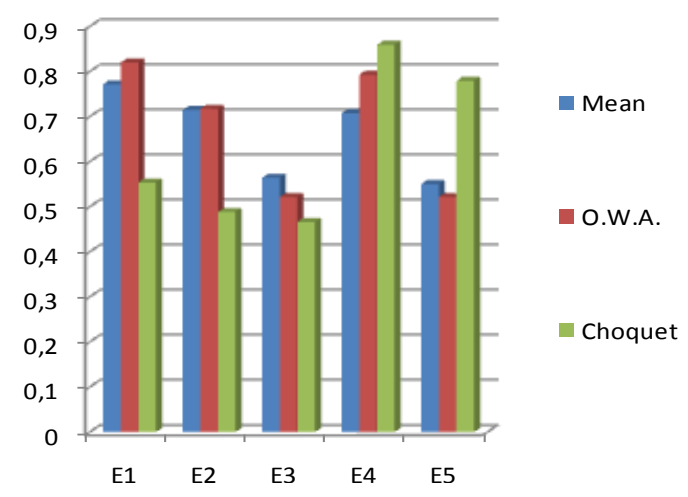

Figure 4: Comparison of health states using different aggregation operators

We will first consider E1 and E2. Those two examples present relatively strong signs of degradation (on torque for E1 and on speed for E2). However, one can see that the Choquet integral reflects better the situation by resulting in a weaker health state than the other operators (22\% to $27 \%$ weaker). This comes from the fact that, according to the expert, both of torque and speed are important.,So, a good value, of one of these indicator, cannot compensate a weak value of the other one.

Then we want to consider E2 and E3. We think those examples should have similar health states because: E2 presents a pronounced degradation, but with a distant next scheduled maintenance operation (so one should have more confidence in the system); and: E3 shows a less pronounced degradation of the system with a closer next scheduled maintenance operation.

The differences of health state regarding E2 and E3 are as follow: $15 \%$ for the mean operator, $19 \%$ for O.W.A. and $2.3 \%$ for Choquet integral.

Therefore, the Choquet integral results are closer to what we expect than the other operators.

Finally we will discuss E4 and E5. The degradations on these examples do not affect the production of the system (torque and speed are within expectations). Hence, health state should be good in a global manner.

As one can see, the Choquet integral results are higher (from $6 \%$ to $25 \%$ ) than the other operators, thus underlying the fact that the system performances are good in a global manner. Moreover, the drift in time of this degradation comes with a decrease of the health state value from $15 \%$ to $27 \%$ for the mean and O.W.A. operators against $8 \%$ of decrease for the Choquet integral. This reflects the preference of the expert to give less importance to aging indicators when performance ones are within expectations. 


\section{Conclusion}

The building of a single indicator, representing a system health, aiming at easing decision making has been presented. This system health indicator is based on the aggregation of several sub-system/equipment functional and dysfunctional indicators. The use of the Choquet integral, as an aggregation operator, makes possible to represent the health state of a system with respect to the expert knowledge and allows considering interactions between indicators. Thus, for instance, a loss of performance on energy consumption will not have the same impact on health state than a loss of performance on production. Whereas, a loss of consumption combined with degradations will have a greater impact. As shown on an example, it enhances the relevancy of the system health indicator vs. mean or OWA operators.

Further academic developments will concern addition or suppression of indicators. Indeed, the coefficients of the capacity being identified for a given number of indicators, addition or subtraction of information will change the point of view of the expert on the system. The development concerning the sensitivity of interaction's importance will help making behavior of the Choquet integral even more similar to the system state behavior. Moreover, it would be appropriate to quantify, or qualify, the level of confidence that one can have in these aggregated values with respect to the number of indicators used. For instance, one cannot have the same confidence in a health assessment based on one indicator, than in a health assessment based on several indicators taken from every functional flow.

Since the aim of the project deals with industrial requirements, a main issue will be to adapt this approach to industrial cases, dealing for instance with hundreds of equipment indicators sampled at different frequency (1 sample per day up to 1000 's per second). This issue is tackle within the project in association with PREDICT company (http://www.predict.fr) specialized in the field of Intelligent Maintenance System. PREDICT develops software applications on monitoring, diagnosis and prognosis in the field of P.H.M. and Proactive facility fleet monitoring and management.

\section{Acknowledgment}

This work is a part of the BMCI project. The authors would like to thank all the partners of the BMCI project: DCNS, CMR, ECM, ECT, EDF, FEMTO, LEAT, LSIS, PREDICT, SIMB. The BMCI project is funded by: CG06, CG13, CG83, CR PACA, FUI, MPM, TPM.

\section{References}

[1] J. Gu, D. Lau and M. Pecht, Health assessment and prognostics of electronic products, in $8^{\text {th }}$ International Conference on Reliability, Maintainability and Safety, 912--919, Chengdu, China, 2009.

[2] R. Callan, B. Larder and J. Sandiford, An Integrated Approach to the Development of an Intelligent
Prognostics Health Management System, in Aerospace Conference, Big Sky, MT, 2006.

[3] A. Volponi, Data Fusion for Enhanced Aircraft Engine Prognostics and Health Management, NASA Contractor Report, CR-2005-214055, 2005.

[4] J-B. Léger, Contribution Méthodologique à la Maintenance Prévisionnelle des Systèmes Industriels de Production: Proposition d'un Cadre Formel de Modélisation. Ph. D. Thesis. Université de Nancy, 1999.

[5] M. Walker and R. Kapadia, Integrated Design of On-line Health and Prognostics Management, in Annual Conference of the Prognostics and Health Management Society, San Diego, CA, 2009.

[6] B. Bouchon-Meunier, Aggregation and Fusion of Imperfect Information, 1st ed, 1995.

[7] M. Grabisch, Evaluation subjective, Concepts et Méthodes pour l'Aide à la Décision, 175--232, 2006.

[8] M. Arif, S. Hafid, T. Brouard and N. Vincent, AWFO (un opérateur d'agrégation) pour la reconnaissane des forms, in $15^{e}$ Congrès francophone AFRIF-AFIA, Reconnaissance des Formes et Intelligence Artificielle (RFIA), Tours, France, 2006

[9] R.A. Yager, On ordered weighted averaging operators in multicriteria decision making, IEEE Transaction on Systems, Man and Cybernetics, 18:183-190, 1988.

[10] M. Grabisch, The application of fuzzy integrals in multicriteria decision making, in European Journal of Operational Research, 89:445--456, Elsevier, 1995.

[11] M. Grabisch, I. Kojadinovic, P. Meyer, A review of methods for capacity identification in Choquet integral based multi-attribute utility theory Applications of the Kappalab R package, in European Journal of Operational Research, 186:766-785, Elsevier, 2007.

[12] M. Grabisch, A new algorithm for identifying fuzzy measures and its application to pattern recognition, in Int. Joint Conf of the $4^{\text {th }}$ IEEE Int. Conf. on Fuzzy System and the $2^{\text {nd }}$ Int. Fuzzy Engineering Symposium, 145--50, Yokohama, Japan, 1995.

[13] J-L. Marichal, M. Roubens, Determination of weights of interacting criteria from a reference set, in European Journal of Operational Research, 124:641--650, Elsevier, 2000

[14] Z. Xu, An Overview of Methods for Determining OWA Weights, International Journal of Intelligent Systems, 20:843--865, 2005.

[15] L.A. Zadeh, A computational approach to fuzzy quantifiers in natural languages, Computers \& Mathematics with Application, 9:149--184, 1983. 